\title{
QUALIDADE DE VIDA DE CLIENTES EM HEMODIÁLISE E NECESSIDADES DE ORIENTAÇÃO DE ENFERMAGEM PARA O AUTOCUIDADO
}

\author{
Quality of life of clients on hemodialisys and needs of nursing guidance for self-care \\ Calidad de vida de clientes en hemodiálisis y necesidades de orientación de enfermería \\ para el autocuidado
}

Iraci dos Santos ${ }^{1}$

Renata de Paula Faria Rocha

Lina Márcia Miguéis Berardinelli ${ }^{3}$

\begin{abstract}
RESUMO
Este trabalho relaciona necessidades de orientação de enfermagem com a qualidade de vida de clientes com doença renal crônica, em hemodiálise, considerando conceitos de Autocuidado de Orem. Método descritivo, mediante entrevista com 43 clientes de um Hospital Universitário do Rio de Janeiro, de 2008 a 2009. Os sujeitos de pesquisa têm hipertensão arterial, encontrando-se 83,72\% em hemodiálise há menos de um ano. Aplicando o Kidney Disease Quality of Life Short Form, obtiveram-se os menores escores nas dimensões: física; emocional; condição de trabalho; capacidade funcional. Esses resultados foram relacionados aos obtidos com as necessidades de orientação de enfermagem para nutrição, ingestão de líquidos, complicações da hemodiálise, anticoagulação, atividade física, de lazer e associação a grupos, concluindo-se que esses clientes encontram-se no Sistema de Autocuidado totalmente compensatório. Sugere-se o desenvolvimento da orientação de enfermagem para 0 autocuidado, em consulta de enfermagem, visando promover a qualidade de vida dos clientes.
\end{abstract}

Palavras-chave: Enfermagem. Autocuidado. Hemodiálise. Qualidade de Vida.

\begin{abstract}
This work relates the needs of nursing guidance with quality of life of patients with chronic kidney disease on hemodialysis, considering conceptions of the Self-care of Orem. Descriptive search, through the interview held with 43 clients of the University Hospital of Rio de Janeiro, from 2008 to 2009 . The Clients has arterial hypertension, being $83.72 \%$ on this treatment for less than a year. Applying the Kidney Disease Quality of Life Short Form obtained the lowest scores in the dimensions: physical emotional, work conditions, functional capacity. These results were related to the ones obtained with the needs of a guidance of nursing for nutrition, fluid intake, hemodialysis complications, anticoagulation, physical and leisure activities with membership associated groups. It was concluded that these clients are in this Totally Compensatory System of Self-care. Then It is suggested the development of the nursing guidance for the self-care through nursing consult, in order to promote the quality of life of the clients.
\end{abstract}

Keywords: Nursing. Self-care. Hemodialysis. Quality of life.

\section{Resumen}

Este trabajo relaciona necesidades de orientación de enfermería con la calidad de vida de clientes con enfermedad renal crónica, en hemodiálisis, considerando conceptos del Autocuidado de Orem. Investigación descriptiva, mediante entrevista con 43 clientes del Hospital Universitario de Rio de Janeiro, de 2008 a 2009. Los sujetos tienen hipertensión arterial, 83,72\% de ellos están en terapia renal hace menos de un año. Aplicando el Kidney Disease Quality of Life Short Form, se obtuve los mejores escores en las dimensiones: física; emocional; condición de trabajo; capacidad funcional. Eses resultados fueron relacionados a los obtenidos con las necesidades de orientación de enfermería para nutrición, ingestión de líquidos, complicaciones con la hemodiálisis, anticoagulación, actividad física, diversiones y asociación a grupos, Se concluyó que eses clientes se encuentran en el Sistema de Autocuidado con total compensación de la enfermera. Se sugiere el desarrollo de la orientación de enfermería para el autocuidado, en consulta de enfermería, para promoción de la calidad de vida de los clientes.

Palabras clave: Enfermería. Autocuidado. Hemodiálisis. Calidad de vida.

\footnotetext{
'Doutora em Enfermagem. Professora Adjunta do Programa de Pós-Graduação da Faculdade de Enfermagem da UERJ. Líder do Grupo de Pesquisa CNPq "Concepções teóricas para o cuidar em saúde e enfermagem". Bolsista de Produtividade em Pesquisa do CNPq. Rio de Janeiro-RJ. Brasil. Email: iraci.s@terra.com.br, ${ }^{2}$ Enfermeira Especialista em Nefrologia. Mestre pelo Programa de Pós-Graduação da Faculdade de Enfermagem da Universidade do Estado do Rio de Janeiro. Professora Assistente da Universidade de Várzea Grande. Cuiabá -MT. Brasil. Email: rpfrocha@yahoo.com.br, ${ }^{3}$ Doutora em Enfermagem. Professora Adjunta do Departamento Enfermagem Médico Cirúrgica da Faculdade de Enfermagem da UERJ. Coordenadora do Núcleo de Extensão da FENF/UERJ. Rio de Janeiro-RJ. Brasil. Email: I.m.b@uol.com.br
} 


\section{INTRODUÇÃO}

A prática do cuidar de clientes com doença renal crônica (DRC), necessitando hemodiálise, é um desafio para a enfermagem. Esse problema caracteriza uma fase de vida de uma pessoa que era saudável, aparentemente sem necessidade de orientações e cuidados de saúde, e passa a depender do atendimento constante e permanente de um serviço de saúde, de uma máquina para desenvolver a tecnologia dialítica administrada por uma equipe multiprofissional.

Nesse sentido, a pessoa toma consciência da perda de autonomia para viver no mundo, consigo, com os outros, perdendo a esperança na continuidade de sua própria vida. Isso porque as doenças crônicas se caracterizam pelo fato de não terem cura ou serem de duração prolongada, e, a longo e/ ou curto prazo, conduzem à morte. A experiência no cuidar desses clientes tem demonstrado que a adaptação às características da DRC constitui um processo extremamente complexo, com sérias implicações e repercussões de variadas ordens.

Considerando ser imprescindível investir na sobrevida do ser humano, propiciando seu bem-estar, independente da sua convivência com essa patologia, neste trabalho indaga-se: qual é a qualidade de vida de clientes com DRC submetidos à terapia de hemodiálise? Diante do exposto, tem-se como objetivo: identificar a qualidade de vida de pessoas com doença renal crônica, em terapia de hemodiálise, aplicando 0 questionário Kidney Disease Quality of Life Short Form, relacionando-a às necessidades de orientação de enfermagem para o autocuidado.

Registra-se que este trabalho é um recorte de Dissertação de Mestrado em Enfermagem, 1 vinculada ao Projeto de Bolsa de Produtividade em Pesquisa do CNPq "Evidências da orientação de enfermagem para o autocuidado na qualidade de vida das pessoas. Com sua realização, espera-se contribuir para diagnosticar a qualidade de vida (QV) do cliente com DRC em tratamento hemodialítico, pois a pessoa com limitações causadas pela enfermidade e pelo tratamento precisa de ações que promovam adaptação ao seu novo estilo de vida e facilitem o enfrentamento dessa patologia e da rotina da terapia renal substitutiva.

\section{REVISÃO DE LITERATURA}

Foram encontrados diversos estudos utilizando questionários para mensuração da qualidade de vida de clientes com DRC. No Brasil, é utilizado o questionário Short Form-36 (SF-36), um instrumento genérico, cujas conclusões dos estudos demonstram sua utilidade para avaliar a QV na situação descrita. Exemplificando, cita-se o estudo que analisou a QV de indivíduos com diferentes tempos de hemodiálise, demonstrando a clara redução dessa qualidade, associada à presença da doença crônica, apontando a necessidade de um tratamento contínuo por um longo período, a idade avançada dos clientes e a presença de comorbidades. ${ }^{2}$

Comparando dois instrumentos genéricos: o WHOQOLbreve e o SF-36 para avaliar a QV da pessoa idosa em hemodiálise, verificou-se que o primeiro apresentou melhor desempenho, sendo que ambos apresentaram elevado nível de confiabilidade. Entretanto, o WHOQOL -breve exibiu maior poder de discriminação. ${ }^{3}$

Assim, constatou-se que ambos os instrumentos mostraram-se confiáveis e válidos para serem empregados na medida da QV dos sujeitos avaliados. Aplicando o SF-36 para verificar se o nível de qualidade de vida associa-se com a duração, frequência de hospitalização e ocorrência de óbito aos clientes com DRC submetidos à hemodiálise, concluiu-se que menores pontuações referentes ao componente físico do SF-36 associaram-se com aumento de hospitalização nesses indivíduos. ${ }^{3}$

Utilizando o mesmo questionário, identificaram-se atividades cotidianas comprometidas após o início da hemodiálise, constatando-se prejuízo da QV dos clientes, os quais apresentaram menores escores nos domínios físicos, emocionais e vitalidade. ${ }^{4}$ Quanto às atividades físicas e recreativas, os pesquisadores relataram os maiores percentuais de comprometimento, referentes a alterações nas atividades domésticas, trabalho e nas atividades práticas, ressaltando-se a importância de o enfermeiro avaliar a QV dos clientes com DRC e promover transformações condizentes com a realidade do indivíduo. ${ }^{5}$

Em busca na base de dados Medical Literature Analysis and Retrieval System Online (MEDLINE), via Biblioteca Virtual em Saúde (BVS), foram encontrados 16 trabalhos utilizando o questionário Kidney Disease and Quality-of-Life-Short Form (KDQOL-SF), porém não foram encontrados trabalhos publicados no Brasil, nem pela enfermagem. Um desses trabalhos, realizado em 2007, utilizou o questionário $K D Q O L$ para avaliar a alteração na QV de pessoas com DRC, em hemodiálise, realizando programa de exercício físico, de baixa intensidade, nas primeiras duas horas de diálise. Observou-se que a execução de atividade física durante a hemodiálise aumentou a eficácia da diálise e a função física dos clientes. ${ }^{1,6}$

Ainda avaliando a QV de clientes com essa patologia e utilizando o mesmo instrumento, observou-se que existiam poucos estudos específicos para a DRC. Os pesquisadores constataram a relação entre a perda da função renal com diminuição nos níveis de QV, enfatizando a necessidade da implementação de políticas de saúde para essa população. ${ }^{7}$ Enquanto, comparando fatores psicossociais com qualidade de vida de clientes em hemodiálise, concluiu-se que a ansiedade e a depressão influenciam fortemente a qualidade de vida de clientes utilizando essa terapia. ${ }^{8}$ 


\section{Um resumo da Teoria de Autocuidado de Dorothéa Orem}

Considerando uma condição fisiológica tal como o acometimento de doenças, o ser humano pode ser incentivado para adquirir novos conhecimentos e habilidades, que o leve a se adaptar às situações, que lhe são impostas e retomar sua vida. Na teoria de Orem, a ação orientada, tomada pelo indivíduo a fim de regular os fatores que afetam seu próprio desenvolvimento, executando atividades que promovam seu bem-estar, sua saúde e sua vida, pode ser caracterizada como ações de autocuidado. ${ }^{9}$

Essa teórica contribuiu na formação de um corpo de conhecimento de enfermagem. Em sua concepção, o cuidado é próprio da ação positiva que tem uma prática e um caminho terapêutico, visando manter o processo da vida e promoção da saúde. 0 cuidado ajuda o indivíduo a crescer, se desenvolver, e também na prevenção, controle e cura de processos de enfermidades e danos. Assim, o autocuidado é a capacidade que os indivíduos têm de cuidar de si mesmos, desempenhando atividades em seu próprio benefício, para manter a vida, a saúde e o bem-estar próprios. ${ }^{9}$

A teoria do autocuidado engloba o conceito, as atividades, a exigência terapêutica e os requisitos para 0 autocuidado. São três os requisitos ou exigência, apresentados por Orem: universais, de desenvolvimento e de desvio de saúde. A demanda de autocuidado terapêutico engloba as ações necessárias para manter a vida e promover a saúde e 0 bemestar. Na teoria de Orem, caso a demanda do autocuidado terapêutico exceda a capacidade de autocuidado do indivíduo, temos a caracterização do déficit de autocuidado, onde se insere a atuação da enfermagem.

A teoria do déficit de autocuidado proporciona um sistema geral para dirigir as atividades de enfermagem, quando as exigências de autocuidado são maiores do que as capacidades para o autocuidado. Na teoria de sistemas, são considerados três tipos de sistemas de enfermagem: 0 autocuidado que se realiza para suprir o que não pode ser realizado pelo cliente - totalmente compensatório; o que atende $o$ indivíduo através do autocuidado - parcialmente compensatório; e o que educa e apoia o indivíduo para ajudá-

\section{METODOLOGIA}

Método descritivo com abordagem quantitativa, transversal e naturalista, visando levantar informações de pessoas sobre determinados assuntos. ${ }^{11}$ Após aprovação do Comitê de Ética em Pesquisa, mediante o Parecer 2172-CEP/ HUPE, a pesquisa foi realizada na Unidade de Diálise da Enfermaria de Nefrologia do Hospital Universitário Pedro Ernesto (HUPE/UERJ), que funciona em três turnos por dia e eventualmente em quatro, dependendo da demanda de clientes.

Participaram da investigação 43 clientes que atenderam aos critérios de inclusão/exclusão: de inclusão-Ter doença renal crônica de qualquer etiologia e encontrar-se em programa de hemodiálise no HUPE/UERJ; com capacidade de compreensão preservada; ambos os sexos com idade $>16$ anos; possuir um contato telefônico e ou e-mail para possíveis comunicações sobre a pesquisa e concordar em participar da pesquisa, assinando o Termo de Consentimento Livre e Esclarecido. Ressalta-se que essa amostra não foi randomizada, devido à decisão de entrevistar todos os clientes em terapia de hemodiálise, no período diurno e noturno, de agosto de 2008 a maio de 2009, reservado para a produção de dados.

\section{Técnica de pesquisa, instrumentos e estratégia de produção de dados}

Para a coleta de dados, a pesquisadora procedeu à entrevista estruturada e individual, com os sujeitos de pesquisa, inicialmente utilizando um formulário para levantamento das necessidades de autocuidado, criado pelas pesquisadoras e validado por especialistas em assuntos referentes a terapias renais substitutivas, qualidade de vida, autocuidado e teoria de Orem, centrado na terapia de hemodiálise, questionando-se: significado da hemodiálise, alimentação, hidratação, acesso venoso, prevenção de complicações, sintomas pós-hemodiálise, controle dos sintomas, necessidades gregária e de relacionamento interpessoal.

Em seguida, aplicou-se o ICD Kidney Disease Quality of Life Short Form (KDQOL-SF) como questionário para mensurar a QV dos clientes em programa hemodialítico. Esse questionário foi traduzido para português em 2003, e sua validação foi realizada em 2005. ${ }^{12}$ Os escores dos itens do KDQOL-SF variam entre 0 e 100; os valores menores correspondem à QV relacionada à saúde menos favorável, enquanto os escores mais elevados refletem maior qualidade de vida.

0 short form-36 (SF-36) é um questionário de saúde genérico que contém 36 questões divididas em 9 domínios: 1) atividade física, 2) atividade social, 3) limitações pela incapacitação física, 4) limitações pela capacidade emocional, 5) saúde mental, 6) vitalidade, 7) dores, 8) percepção da saúde em geral e 9) percepção da melhora do problema específico na saúde. A pontuação varia de 0 a 100, e o maior número significa melhor saúde e menos dor.

0 KDQOL-SF é um instrumento específico que avalia DRC, em fase terminal. É autoadministrável e contém 80 itens. Ele inclui o 36 Item Short-Form Health Survey (SF-36) como uma medida genérica, sendo suplementado com escalas do tipo multi- itens, voltadas para as preocupações particulares dos clientes com DRC. É considerado o questionário mais completo disponível atualmente para avaliar a QV de clientes com a citada patologia. ${ }^{13}$

Em suplemento ao SF-36, há uma escala multi-itens abordando indivíduos com DRC em diálise, incluindo itens direcionados à doença renal: sintomas/problemas físicos (12 itens), efeitos da doença renal em sua vida diária (8 itens), sobrecarga 
imposta pela doença renal ( 4 itens), condição de trabalho ( 2 itens), função cognitiva (3 itens), qualidade das interações sociais ( 3 itens), função sexual (2 itens) e sono (4 itens). Inclui ainda, respectivamente, dois itens de suporte social e sobre apoio da equipe profissional de diálise e um item sobre satisfação do cliente. A avaliação da saúde geral (1 item) é computada à parte.

A produção de dados foi realizada durante a hemodiálise, por ser um período em que, na maioria das vezes, o cliente está ocioso, ligado à máquina por um tempo médio de quatro horas, sendo esta uma oportunidade de preencher esse tempo com alguma atividade. Assim utilizou-se de 30 a 40 minutos para a aplicação dos instrumentos de produção de dados.

Para análise dos dados referentes à identificação de necessidades de autocuidado, foi utilizada estatística descritiva simples, considerando um formulário de 11 questões com pontuação variando de 3 a 10 pontos. Foi estabelecido um Critério de Avaliação a partir das concepções da Teoria de Sistemas de Autocuidado de Orem:-10 Sistema totalmente compensatório, em que o cliente é incapaz de empenhar-se nas ações de autocuidado; Sistema parcialmente compensatório, no qual o enfermeiro e o cliente executam medidas ou ações para o autocuidado; e o Sistema apoio-educação, no qual o cliente consegue executar o autocuidado, sendo assim suas exigências resumem-se a tomada de decisões, controle do comportamento e aquisição de conhecimentos e habilidades. Para cada sistema foi atribuído um valor e conceito, a saber: 100-80-Conceito A - Sistema apoio-educação; 79-50-Conceito B - Sistema parcialmente compensatório; abaixo de 50 Conceito C - Sistema totalmente compensatório.

A pontuação de cada questionário foi lançada em um banco de dados, tendo-se utilizado estatística descritiva e medidas de tendência central: média e desvio-padrão. Os dados do questionário de qualidade de vida, KDQOL-SF, foram analisados utilizando-se o programa de análise disponibilizado pelo KDQOL-SF Working Group.

\section{RESULTADOS}

Os resultados encontrados com a aplicação do questionário de qualidade de vida KDQOL-SF apresentam-se na Tabela 1.

Tabela 1.Média e desvio-padrão das dimensões do KDQOL-SF entre clientes em terapia de hemodiálise. N-43. Rio de Janeiro, HUPE/UERJ, 2009.

\begin{tabular}{lcc}
\multicolumn{1}{c}{ DIMENSÕES (número de itens) } & MÉDIA & DESVI0-PADRÃO \\
\hline Sintomas / problemas (12) & 77,52 & 18,01 \\
Efeitos da doença renal (8) & 55,96 & 23,22 \\
Sobrecarga imposta pela doença renal (4) & 33,72 & 24,86 \\
Condição de trabalho (2) & 9,3 & 25,01 \\
Função cognitiva (3) & 86,36 & 23,27 \\
Qualidade das interações sociais (3) & 78,29 & 27,45 \\
Função sexual (2) & 75 & 43,3 \\
Sono (4) & 72,73 & 27,74 \\
Suporte social (2) & 76,74 & 29,13 \\
Apoio da equipe de diálise (2) & 57,56 & 32,86 \\
Satisfação do paciente (1) & 55,81 & 17,01 \\
Capacidade funcional (10) & 33,37 & 28,02 \\
Limitações causadas por problemas da saúde física (4) & 8,72 & 23,71 \\
Dor (2) & 80,23 & 33,2 \\
Percepções da saúde geral (5) & 41,16 & 21,6 \\
Bem-estar emocional (5) & 67,26 & 30,9 \\
Limitações causadas por problemas da saúde emocional(3) & 15,5 & 33,61 \\
Função social (2) & 61,92 & 35,03 \\
\hline Sitalidade (energia/fadiga) geral (1) & 53,84 & 29,01 \\
\hline & 56,98 & 2,31 \\
\hline
\end{tabular}

Legenda: negrito= quantitativo de problemas destacando dificuldades para a QV do cliente 
Observam-se nessa tabela as seguintes dimensões com menores escores: limitações causadas por problemas da saúde física $(8,72 \pm 23,71)$; condição de trabalho $(9,3 \pm 25,01)$; limitações causadas por problemas da saúde emocional (15,5 $\pm 33,61)$; capacidade funcional $(33,37 \pm 28,02)$ e sobrecarga imposta pela doença renal ( $33,72 \pm 24,86)$.

Resultados semelhantes foram encontrados nos estudos. ${ }^{1415}$ No primeiro ${ }^{14}$, foram encontrados menores escores nas dimensões condição de trabalho, função física e sobrecarga de doença renal, enquanto as dimensões suporte social, estímulo por parte da equipe de diálise e função cognitiva apresentaram maiores escores médios. 0 segundo ${ }^{15}$ relatou menores valores nas dimensões sobrecarga da doença renal, condição de trabalho e funções sexual, física e emocional.

Quanto às limitações causadas por problemas da saúde física, a DRC causa uma série de restrições e prejuízo nos estados de saúde física, funcional, bem-estar geral, interação social e satisfação dos clientes interferindo diretamente na qualidade de vida. ${ }^{4-5}$ Normalmente, as pessoas conceituam saúde como ausência de doença ou sintomas. Sendo assim, 0 cliente com DRC considera-se sempre doente e isso interfere no seu bem-estar. ${ }^{1}$

0 cliente com DRC apresenta limitações físicas para andar, realizar esforço físico, carregar peso, subir escadas, correr, entre outras. Assim, percebe-se que eles devem adaptarse a várias mudanças. A maneira de reagir diante da doença difere de indivíduo para indivíduo. Entretanto, a necessidade de reaprender a viver é coerente indispensável ao ser humano. ${ }^{1,3-6}$ Esse processo de aceitação de sua condição interfere na qualidade de vida das pessoas. A adaptação não acontece em um passe de mágica, é um processo complexo que mobiliza estruturas individuais. ${ }^{1,16}$

Ressalta-se um estudo mostrando que os clientes em terapia de hemodiálise apresentaram desestruturação quanto ao funcionamento ocupacional nos aspectos relativos à organização do tempo, flexibilidade, mudanças da rotina, objetivos e expectativas para o futuro. ${ }^{16}$ No entanto, é importante lembrar que as pessoas em hemodiálise não ficam totalmente impossibilitadas de realizar as tarefas do seu cotidiano. Os autores concluem que, mesmo diante das dificuldades, com o cotidiano alterado por horários de terapia, restrições alimentares, e, em muitos casos, a perda do trabalho e/ou do estudo, os indivíduos com DRC podem, com ajuda de profissionais: planejar atividades, construir projetos e, assim, fazer algo com significado para si, reconstruindo seu cotidiano. ${ }^{14,16}$

Outro fator que interfere na QV do cliente com DRC é a modificação dos hábitos alimentares e a restrição hídrica. Percebe-se que isso surge com as limitações, pois é necessária a adoção de uma dieta com diminuição da ingestão proteica, sódio, potássio e água. ${ }^{5,16}$ Esse é um desafio a ser enfrentado, pois cada um comete transgressões por motivos diferentes, e a cultura e os hábitos da família podem marginalizar o cliente, considerando que sua dieta é preparada separadamente, ${ }^{14,16-}$ 17 o que gera reclamações e o sentimento de exclusão no cliente.

A doença e o tratamento geralmente surgem de forma abrupta e inesperada, gerando muita tristeza nos clientes que necessitam adaptar-se às mudanças causadas. Como intercorrências do procedimento de hemodiálise, destacamse a hipotensão, cãibras, vômitos, cefaleia, mal-estar, entre outros. ${ }^{1}$ A autoimagem também é alterada pela necessidade de instalação de uma via de acesso para a diálise, ou seja, a criação de uma fístula arteriovenosa (FAV) ou a inserção de um cateter. ${ }^{17}$ Além disso, esses acessos exigem uma série de cuidados tanto para o cliente como para os profissionais que os implementam, devido aos riscos de contaminação por agentes biológicos. ${ }^{18}$

0 baixo escore na dimensão condição de trabalho demonstra o iminente problema relacionado à vida profissional, na qual a maioria das pessoas com DRC, em terapia de hemodiálise, não consegue estabelecer e/ou manter vínculo com trabalho, provavelmente devido ao tempo dedicado ao tratamento, presença de queixas físicas de fraqueza, cansaço, indisposição, mal-estar geral, nos dias de realização de hemodiálise. Isso impossibilita a realização de esforço físico para o trabalho. Recorda-se, que o próprio mercado de trabalho, no Brasil, oferece poucas oportunidades para as pessoas com limitações dessa natureza. ${ }^{1}$

A maioria dos clientes tem baixo nível de escolaridade tornando-se difícil ou inviável o exercício profissional, em uma sociedade capitalista com mercado de trabalho competitivo. Destaca-se que o trabalho para as pessoas representa sua própria vida, possibilita sua autorrealização profissional, bem como a autonomia financeira para manutenção da instituição familiar, principalmente para os adultos. Entretanto, a DRC e os seus tratamentos não caracterizam impedimento direto e absoluto ao trabalho, mas causam limitações importantes às pessoas, muitas vezes ocasionando afastamentos e aposentadorias dela decorrentes. ${ }^{6,17}$

Muitas vezes, o cliente é a principal fonte de renda da família. Com a evolução da DRC, ocorre um afastamento de suas atividades (de trabalho e/ou de estudo), idas frequentes ao hospital, consultas médicas, internações, entre outros, em decorrência do próprio tratamento. Portanto, torna-se indispensável o redimensionamento do orçamento familiar para enfrentar as interferências da doença nas atividades de trabalho e de estudo da pessoa. 0 enfermeiro pode atuar nessa fase orientando que 0 afastamento pode ser temporário. ${ }^{1,17}$

Ao restabelecer-se de uma fase aguda, esse cliente pode realizar atividades laborais/de estudo. Se não for possível continuar as atividades anteriores, ele pode engajar-se em atividades alternativas. A pessoa para considerar-se capaz, deve ser estimulada a adaptar-se à situação atual, buscando soluções adequadas à sua condição. A busca da qualidade de vida não está em fazer tudo que era feito antes da doença, e 
sim em adaptar-se à atual situação e buscar satisfação dentro das atividades realizadas. ${ }^{1,17}$

Os clientes que possuem vínculo com trabalho provavelmente são mais independentes e possuem maior autoestima e autorrealização, e assim manifestam maior satisfação com o tempo e apoio que recebem de familiares e amigos. Já os clientes que não conseguem estabelecer e/ou manter esse vínculo com o trabalho têm a sensação de frustração e impotência, além de serem mais dependentes, levando à manifestação de insatisfação com o tempo e apoio que recebem de familiares e amigos. ${ }^{16,617}$

Na dimensão das limitações causadas por problemas da saúde emocional, pode-se dizer que a DRC, além de ter um impacto grande na parte física, ela também tem influência na parte emocional. A DRC e seu tratamento causam no cliente a perda dos seus sonhos e aspirações, mudança no estilo de vida, perda das escolhas e opções, além do isolamento social. A condição crônica e o tratamento hemodialítico são fontes de estresse e representam desvantagem por ocasionar problemas: isolamento social, perda do emprego, dependência da Previdência Social, parcial impossibilidade de locomoção e passeios, diminuiç̧ão da atividade física, necessidade de adaptação à perda da autonomia, alterações da imagem corporal e, ainda, um sentimento ambíguo entre medo viver e de morrer., ${ }^{6,17}$

Um fator que influencia muito o bem-estar emocional desses clientes é a sensação de dependência. Dependência da família, da equipe de saúde, de uma máquina, de medicamentos. Essa dependência provocando uma realidade, reveladora da inexistência de opção, devido à necessidade do tratamento. Essa obrigatoriedade em aceitá-lo como única forma de manutenção da vida cria uma sensação de perda da liberdade. É nesse sentido que o conviver com a DRC diz respeito à família. 0 fato de receber apoio das pessoas queridas faz o cliente sentir-se melhor., ${ }^{17}$

A dimensão capacidade funcional e a dimensão sobrecarga imposta pela doença renal estão inter-relacionadas e são diretamente dependentes da saúde física, para serem avaliadas pelos clientes. São queixas constantes dos clientes em hemodiálise a falta de energia, sensação de desânimo e fadiga, 0 que provavelmente diminui os escores das dimensões supracitadas. ${ }^{17} \mathrm{~A}$ autora afirma ${ }^{17}$ que o fato de os clientes viverem com um(a) companheiro(a) contribui para o cuidado no domićlio, pois a DRC contribui para perdas funcionais com comprometimento da independência e autonomia, muitas vezes tornando-os dependentes parciais ou totais dos cuidados de outra pessoa.

\section{Relação entre as necessidades de orientação de enfermagem para 0 autocuidado e qualidade de vida dos clientes em hemodiálise}

A QV, avaliada pelo KDQOL-SF, dos clientes com DRC em tratamento hemodialítico, teve como resultados os mais baixos escores médios nas dimensões: limitações causadas por problemas da saúde física; condição de trabalho; limitações causadas por problemas da saúde emocional; capacidade funcional e sobrecarga imposta pela doença renal.

Relacionando esse resultado com o obtido no questionário para avaliação das necessidades de orientação de enfermagem para o autocuidado, tem-se: problemas da saúde física relacionado com terapia nutricional, ingestão de líquidos, complicações da hemodiálise, anticoagulação e prática de atividade física; relacionadas a problemas de saúde emocional tem-se a associação a grupos e a atividades de lazer; e relacionadas à capacidade funcional e sobrecarga da doença renal tem-se de prática de atividade física.

Esses itens possuem importante relação com o resultado obtido no questionário de autocuidado, observandose que $60,47 \%$ dos clientes encontram-se no sistema totalmente compensatório. ${ }^{17}$

À medida que o cliente alcançar independência e autonomia nas ações de autocuidado, essas dimensões afetadas medidas pelo KDQOL sofrerão alteração. A pessoa que conhece o tratamento sabe sobre suas complicações e como preveni-las, evitando o ganho excessivo de peso interdialítico e seguindo as restrições alimentares, bem como cuidar de seu acesso venoso. Ela realiza atividade física, tem bom relacionamento interpessoal, tem atividades de lazer, toma os medicamentos conforme prescrição e realiza o tratamento dialítico conforme prescrição. Tendo, portanto, grandes chances de apresentar altos escores nas dimensões do KDQOL. ${ }^{1}$

Então, apresenta-se um importante desempenho para o enfermeiro, pois ele pode atuar orientando esse cliente para o alcance de independência e autonomia nessas ações de autocuidado, valorizando sua qualidade de vida. 0 enfermeiro exerce papel destacado no tratamento deste cliente, tendo em vista que é o membro da equipe de saúde que pode estar mais próximo ao cliente e à família, exercendo práticas educativas, de cuidados e administrativas. ${ }^{1,17}$

Muitas vezes, o cliente inicia o tratamento dialítico em caráter emergencial; sendo assim, ele não é orientado acerca da doença e do tratamento. 0 cliente precisa ser ajudado, pela equipe de saúde, a compreender o que está acontecendo com ele. Assim, cabe à enfermeira desenvolver estratégias educativas com o intuito de orientá-lo sobre sua enfermidade, manifestações clínicas, estilo de vida saudável, tratamento, cuidados com o acesso venoso, dentre outras temáticas, conforme as reais necessidades do cliente. 0 enfermeiro reconhece o cliente como agente de seu autocuidado, conhecendo seu tratamento e participando da elaboração do seu plano de cuidados. ${ }^{17}$

0 cliente com DRC, principalmente em tratamento hemodialítico, cria laços com a equipe de saúde responsável pelo seu tratamento. 0 convivio frequente que a hemodiálise proporciona é o responsável pelo vínculo. A equipe de saúde deve estar atenta para que esse relacionamento não se transforme em dependência, e sim, em um vínculo terapêutico. 
A formação de laços afetivos, também entre os clientes, estabelece uma moral coesão entre eles. Fato importante para a saúde emocional das pessoas que reflete em sua qualidade de vida. ${ }^{17}$

\section{CONCLUSÃO}

A maioria dos clientes do estudo é incapaz para executar ações de autocuidado e apresenta baixos escores nas dimensões do instrumento, que mede a $Q V$ relacionada às ações de autocuidado. Confirmando ser o KDQOL-SF um instrumento capaz de avaliar a QV de clientes em hemodiálise.

Nesta pesquisa concluiu-se que, por ser a DRC uma doença crônica e não transmissível, a continuidade de seu tratamento afeta vários aspectos da vida dos clientes, sendo assim muito importante a avaliação da sua QV e grandes as necessidades de autocuidado, para que possam ser instituídas ações que melhorem sua sobrevida.

Importa, ainda, ressaltar que a DRC e seu tratamento causam incapacidades físicas e emocionais, interferindo na vida das pessoas, limitando ou impedindo a realização de suas atividades diárias. Os resultados desta investigação confirmam essa afirmação diante da obtenção dos menores escores em limitações causadas por problemas da saúde física; condição de trabalho; limitações causadas por problemas da saúde emocional; capacidade funcional e sobrecarga imposta pela doença renal.

Ressalte-se, que a avaliação da QV por meio do KDQOL-SF auxilia os profissionais de saúde, atuantes em nefrologia a diagnosticar as condições de saúde individuais e/ ou coletivas, e assim subsidiar o planejamento do cuidado. Recorda-se, que uma forma de obtenção de QV do indivíduo com DRC é adiar o início do tratamento dialítico através de ações como diagnóstico precoce de lesão renal, início do tratamento conservador, controle de pressão arterial e glicemia. Porém, diante do quantitativo de aproximadamente 67.000 pessoas, realizando hemodiálise no Brasil, algo tem de ser feito para favorecer sua qualidade de sobrevida.

Com relação ao ensino, a divulgação deste trabalho pode contribuir com a equipe de enfermagem na implementação de um processo de pesquisar/ensinar-cuidar da pessoa, considerando a construção responsável de ações relativas ao seu autocuidado.

Quanto à prática de pesquisa, espera-se contribuir com o desenvolvimento de futuras investigações na área de enfermagem em nefrologia, utilizando a sistematização da assistência para educação à saúde. Do mesmo modo, através dos estudos que avaliam a qualidade de vida das pessoas, sugere-se aprofundar o conhecimento sobre a propriedade de se investir na orientação para autocuidado, durante a consulta de enfermagem aos clientes acometidos de DRC e, principalmente, àqueles participantes de Programas de Hemodiálise.

\section{REFERÊNCIAS}

1. Rocha RPF. Necessidades de orientação de enfermagem para o autocuidado visando a qualidade de vida de clientes em terapia de hemodiálise. [dissertação]. Rio de Janeiro (RJ): Faculdade de Enfermagem,Universidade Estadual do Rio de Janeiro; 2010.

2. Castro OM, et al. Qualidade de vida de pacientes com insuficiência renal crônica em hemodiálise avaliada através do instrumento genérico SF-36. Rev Assoc Med Bras. 2003; 49(3): 245-49.

3. Souza FF. Avaliação da qualidade de vida do idoso em hemodiálise: comparação de dois instrumentos genéricos. [dissertação] Campinas(SP): Faculdade de Ciências Médicas, Universidade Estadual de Campinas; 2004.

4. Martins MRI, Cesarino CB. Qualidade de vida de pessoas com doença renal crônica em tratamento hemodialítico. Rev Latino-am Enfermagem. 2005; 13 (5): 670-76.

5. Santos PR, Pontes LRSK. Mudança do nível de qualidade de vida em portadores de insuficiência real crônica terminal durante seguimento de 12 meses. Rev Assoc Med Bras. 2007; 53 (4): 329-34.

6. Parsons TL et al. Exercise training during hemodialysis improves dialisys efficacy an physical performance. Arch Phys Med Rehabil. 2006; 87 (1): 680-87.

7. Gorodetskaya I, et al. Health-related quality of life and estimates of utility in chronic kidney disease. Kidney Int. 2005; 68 (2): 2801-808.

8. Vasques I, et al. Psychosocial factors and health-related quality of life in hemodialysis patients. Qual Life Res. 2005; 14 (1): 79-190.

9. Santos I dos, Sarat CNF. Modalidades de aplicação da Teoria do Autocuidado de Orem em comunicações científicas brasileiras. Rev Enferm UERJ. 2008: 16 (3):313-18.

10. Eben JD, Gasti NN, Hayes SE, Marriner-Tomey A, Nation MJ, Nordmeyer SB. Dorothea E Oren: Self care deficit theory of nursing. In: MarrinerTomey A, Alligood MR. Nursing theorists and their work. $5^{\text {a }}$ ed. Madrid: Harcourt Brace; 2005. p.181-99.

11. PollitDF, BeckCT, Hungler BP. Fundamentos de pesquisa em enfermagem. $3^{\text {a }}$ ed. Porto Alegre(RS): Arte Médica; 2004.

12. Duarte PS, Cicconelli RM, Sesso R. Cultural adaptation and validation of the Kidney Disease and Quality of Life- Short Form (KDQOL-SF 1.3) in Brazil. Braz J Med Biol Res. 2005; 38(2): 261-70.

13. Duarte OS, et al. Tradução e adaptação cultural do instrumento de avaliação de qualidade de vida para pacientes renais crônicos. Rev Assoc Med Bras. 2003; 49 (4): 375-81.

14. Kusumota L. Avaliação da qualidade de vida relacionada à saúde de pacientes em hemodiálise [tese] Ribeirão Preto (SP): Escola de Enfermagem, Universidade de São Paulo; 2005.

15. Moreira CA, et al. Avaliação das propriedades psicométricas básicas para a versão em português do KDQOL-SF. Rev Assoc Med Bras. 2009; 55 (1): 22-28. 
16. Bezerra KV, Santos JLF. 0 cotidiano de pessoas com insuficiência renal crônica em tratamento hemodialítico. Rev Latino-am Enfermagem. 2008; 16(4): 686-91.

17. Pacheco GS, Santos I. Cuidar de cliente em tratamento conservador para doença renal crônica: apropriação da teoria de Orem. Rev Enferm UERJ 2005; 13 (2): 257-62.

18. Silva MKD, Zeitoune RG. Riscos ocupacionais em um setor de hemodiálise na perspectiva dos trabalhadores da equipe de enfermagem. Esc Anna Nery. 2009; 13 (2): 279-87. 